\title{
Forces and conductances in a single-molecule bipyridine junction
}

\author{
Stadler, Robert; Thygesen, Kristian Sommer; Jacobsen, Karsten Wedel
}

Published in:

Physical Review B Condensed Matter

Link to article, DOI:

10.1103/PhysRevB.72.241401

Publication date:

2005

Document Version

Publisher's PDF, also known as Version of record

Link back to DTU Orbit

Citation (APA):

Stadler, R., Thygesen, K. S., \& Jacobsen, K. W. (2005). Forces and conductances in a single-molecule bipyridine junction. Physical Review B Condensed Matter, 72(24), 241401.

https://doi.org/10.1103/PhysRevB.72.241401

\section{General rights}

Copyright and moral rights for the publications made accessible in the public portal are retained by the authors and/or other copyright owners and it is a condition of accessing publications that users recognise and abide by the legal requirements associated with these rights.

- Users may download and print one copy of any publication from the public portal for the purpose of private study or research.

- You may not further distribute the material or use it for any profit-making activity or commercial gain

- You may freely distribute the URL identifying the publication in the public portal

If you believe that this document breaches copyright please contact us providing details, and we will remove access to the work immediately and investigate your claim. 


\title{
Forces and conductances in a single-molecule bipyridine junction
}

\author{
R. Stadler, K. S. Thygesen, and K. W. Jacobsen \\ Center for Atomic-scale Materials Physics, Department of Physics, Technical University of Denmark, \\ DK-2800 Kongens Lyngby, Denmark
}

(Received 30 September 2005; revised manuscript received 11 November 2005; published 5 December 2005)

\begin{abstract}
Inspired by recent measurements of forces and conductances of bipyridine nanojunctions, we have performed density functional theory calculations of structure and electron transport in a bipyridine molecule attached between gold electrodes for seven different contact geometries. The calculations show that both the bonding force and the conductance are sensitive to the surface structure, and that both properties are in good agreement with experiment for contact geometries characterized by intermediate coordination of the metal atoms corresponding to a stepped surface. The conductance is mediated by the lowest unoccupied molecular orbital, which can be illustrated by a quantitative comparison with a one-level model. Implications for the interpretation of the experimentally determined force and conductance distributions are discussed.
\end{abstract}

DOI: 10.1103/PhysRevB.72.241401

PACS number(s): 73.63.Rt, 73.20.Hb, 73.40.Gk

With the advance of new experimental techniques for the fabrication of atomic-scale contacts, it has become possible in recent years to study electron transport through a few or even a single molecule suspended between metal electrodes. ${ }^{1-4}$ The atomistic details of the local contact geometry are crucial in such experiments, as is evident from the low degree of reproducibility of, for example, the measured $I-V$ curves $^{3}$ or conductance traces. ${ }^{4}$ Since the exact contact geometry is difficult to control and characterize, theoretical calculations are needed for interpreting and understanding the results of the experiments.

Recently, the conductance of bipyridine molecules in a toluene solution trapped between two gold electrodes has been measured simultaneously to the force required to break the contact. ${ }^{5}$ The statistical data derived from $\sim 1000$ independent measurements show a quantization of both the conductance and the rapture force, indicating that the contacts consist of single molecules. Such bipyridine junctions are of interest for two reasons: (i) it is one of the few systems where fairly reliable measurements exist of several properties (forces and conductances) for junctions of single aromatic molecules, and (ii) pyridine contacts represent an alternative to the more commonly used thiol anchor groups. ${ }^{6}$ Conductances for a bipyridine molecule between defect-free
$\mathrm{Au}$ (111) surfaces have recently been calculated ${ }^{7}$ for selected structures using a cluster model for the description of the electrodes. However, in the experiment the surface structures are not known, and here we take up the issues of stability and the extent to which the force and conductance distributions can be related to local structure.

We have performed plane wave-based density functional theory ${ }^{8}$ (DFT) calculations for the conductance and breaking force of a bipyridine contact. The contact between the surface and the molecule is modeled by a variety of different structures based on the (111) orientation of the Au fcc lattice. By varying the substrate-adsorbent distance rigidly we obtain total energy curves, from which rapture forces can be derived for the different contact geometries. A recently developed scheme for coherent electron transport ${ }^{9}$ allows us to calculate the conductance of the molecule within the same theoretical framework as employed for the total energy calculations. Based on our data obtained for the different contact geometries we find that the force and conductance distributions observed in the experiment can only be understood in terms of a high structure selectivity for the contact geometry.

Figure 1 shows an example of the Au-bipyridine-Au nanojunctions and defines the different electrode geometries investigated in this paper. By using our DFT plane-wave
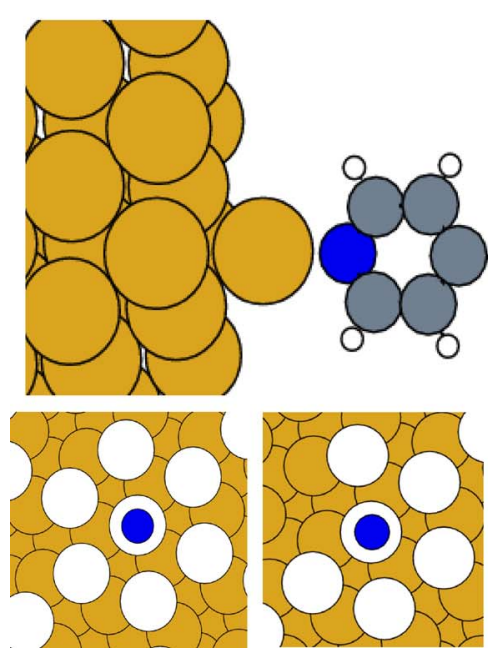

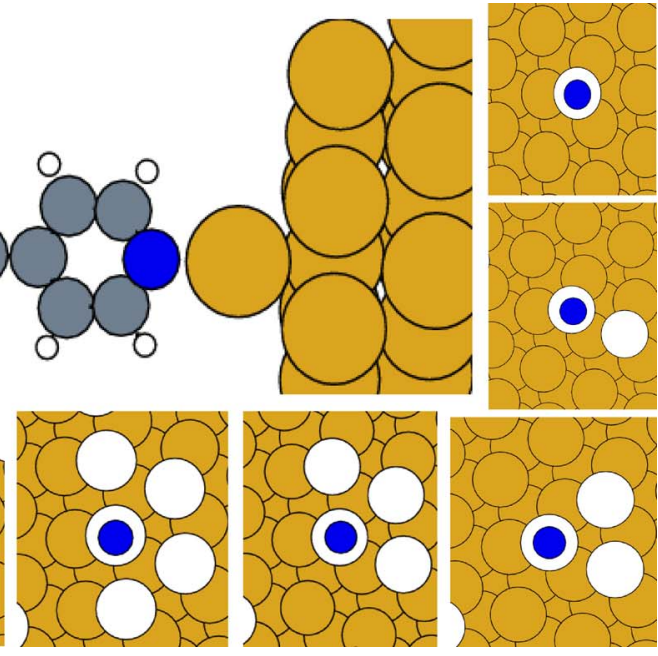

FIG. 1. (Color online) A bipyridine molecule suspended between Au electrodes. The insets illustrate the variation of the surface contact geometry, where clockwise from top right to bottom left a single ad layer with one to seven $\mathrm{Au}$ ad atoms (white spheres), where $N_{c}$ varies from three to nine (see text), on top of a flat $\mathrm{Au}$ (111) surface are shown. Smaller dark spheres define the nitrogen positions. 
approach $^{8}$ with the Perdew-Wang91 (PW91) functional for exchange and correlation ${ }^{10}$ and an energy cutoff of $340 \mathrm{eV}$ for the plane-wave expansion of the wave functions and a cutoff which is four times as high for the charge density, we have established that bipyridine prefers to bond to the $\mathrm{Au}$ (111) surface in an on-top position, and therefore all calculations have been performed for an on-top contact. The supercells consist of repeated slabs with seven Au layers in a (111) stacking with $3 \times 3$ periodicity within the surface plane, where the slabs are linked by one bipyridine molecule per unit cell. As depicted in Fig. 1 the number of Au atoms in the surface plane has been varied from one to seven in such a way that the coordination number $N_{c}$ of the Au atom directly connected to the molecule varies from three to nine. The two structures, where the remaining vacancies in the surface plane are also filled for $N_{c}=9$, are not shown in Fig. 1 , since our calculations showed that occupying these atomic positions has no effect on the forces or conductances. The geometry of the isolated molecule has been relaxed by total energy minimization, but the Au atoms have been fixed to their positions in the bulk crystal structure throughout our study. The torsion angle $\alpha$ which arises if the two pyridine segments within the molecule are rotated relative to each other has been optimized and found to be around $25^{\circ}$ when the molecules are adsorbed in the junction. However, since the energy gain of this rotation is only around $0.03 \mathrm{eV}$, we cannot predict the effect that a solvent or finite temperatures might have on it. We found that the influence of this rotation on the calculated forces is negligible. For the conductance we have performed calculations for both $\alpha=0^{\circ}$ and $25^{\circ}$. An optimization of the Au-N bond length gave values between 2.12 and $2.42 \AA$ depending on the number of atoms in the surface plane but showed no dependency on $\alpha$.

In the top panel of Fig. 2 the binding energies at the optimal Au-N bond lengths for all the structures are shown. The binding energies vary significantly with an almost linear dependence on $N_{c}$, and their magnitude indicate intermediate-strength bonding stronger than typical van der Waals bonding but weaker than covalent bonding between open shell systems. Depending on the detailed choice of the exchange-correlation functional the binding energies may shift a few tenths of an $\mathrm{eV}$ but the trend with the metal coordination number is unchanged (see Fig. 2). The same linear dependence of the binding energy on $N_{c}$ has been found for the chemisorption of oxygen and $\mathrm{CO}$ on Au chains and surfaces, ${ }^{12}$ indicating that the nature of the bonding is also covalent in the present case. A closer inspection of the surface states shows that the trend in the binding energies correlates with an upward shift of the $d$-states towards $E_{F}$ which is expected to enhance the energy gain of a hybridization with the highest occupied molecular orbital [(HOMO), see inset] located at $\sim-4.6 \mathrm{eV} .{ }^{13}$ These findings are in contrast with a recent article, in which the adsorption of pyridine on $\mathrm{Au}$ (111) surfaces was interpreted in terms of Van der Waals interactions ${ }^{6}$ based on more qualitative arguments such as the amount of charge buildup in the center of the bonding region.

From the total energy curves obtained by varying the $\mathrm{Au}-\mathrm{N}$ bond lengths, the breaking forces can be calculated by taking the derivatives at the inflexion point. As can be seen
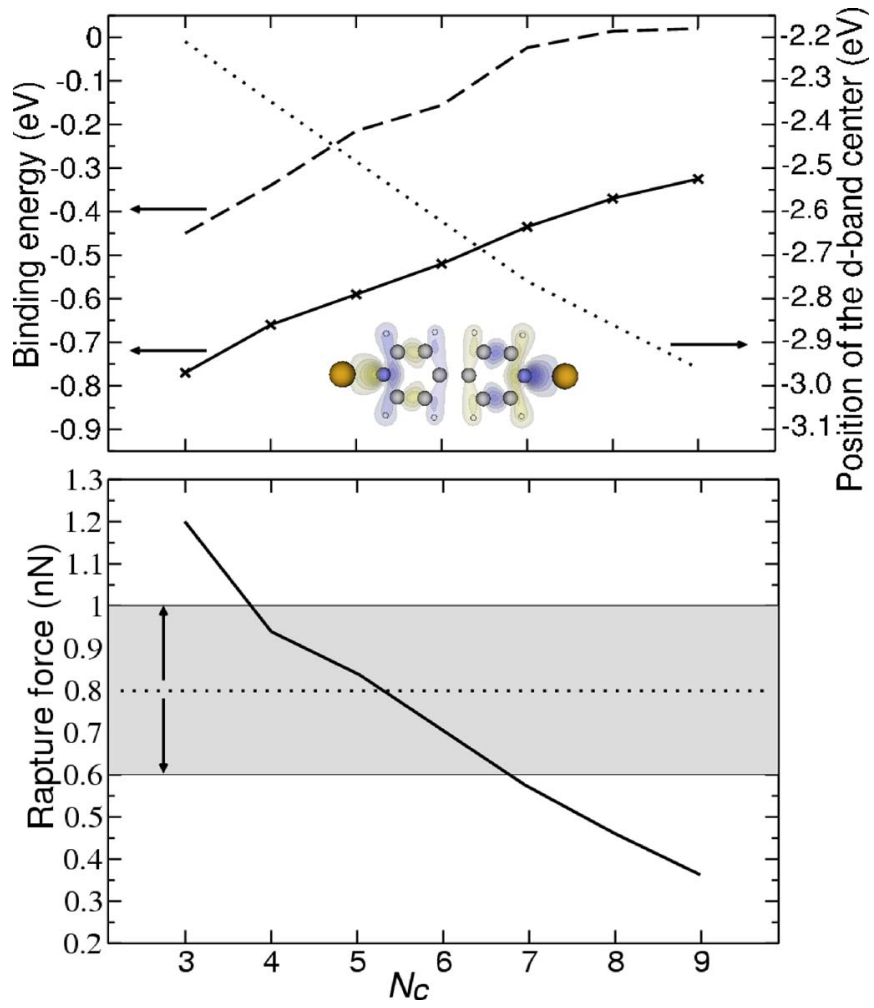

FIG. 2. (Color online) Top: Binding energies for a single N-Au bond as a function of $N_{c}$. The inset shows the HOMO. The solid (dashed) line marks calculations using the PW91 (Ref. 10) (RPBE (Ref. 11)) XC functional. The position of the $d$ band center with respect to the Fermi level is shown as a dotted line. Bottom: Rapture force as a function of $N_{c}$. The horizontal dotted line indicates the experimental value and the gray shaded area marks the region defined by the experimental error bars.

in Fig. 2 the forces like the energies depend almost linearly on $N_{c}$. The rather large variation of the calculated breaking forces should be compared with the experimental situation where a quite sharp peak at a value of $0.8 \pm 0.2 \mathrm{nN}$ is found. ${ }^{5}$ This distribution is consistent with our results if the experimental situation results in the preference for a subset of structures in the range $N_{c}=4-7$. Such a preference could be either thermodynamic or kinetic in origin. Energetically bipyridine clearly favors to bond to metal sites with low metalcoordination numbers according to the calculations. On the other hand such metal defects may be energetically costly to produce and therefore limited in number. Also the experimental process in which the nanocontact is created may bring the system out of local equilibrium and the resulting structures will then depend on the evolution of the process. Finally it should be noted that the calculations do not take potential effects of the solvent into account.

The conductance of the bipyridine junctions is calculated using a numerical method for phase-coherent electron transport which combines a Green's function formalism with a plane wave-based DFT description of the atomic and electronic structure of the entire system. The Green's function of the central region, comprising the molecule and part of the gold electrodes, is evaluated in terms of a basis consisting of maximally localized Wannier functions which are defined by 

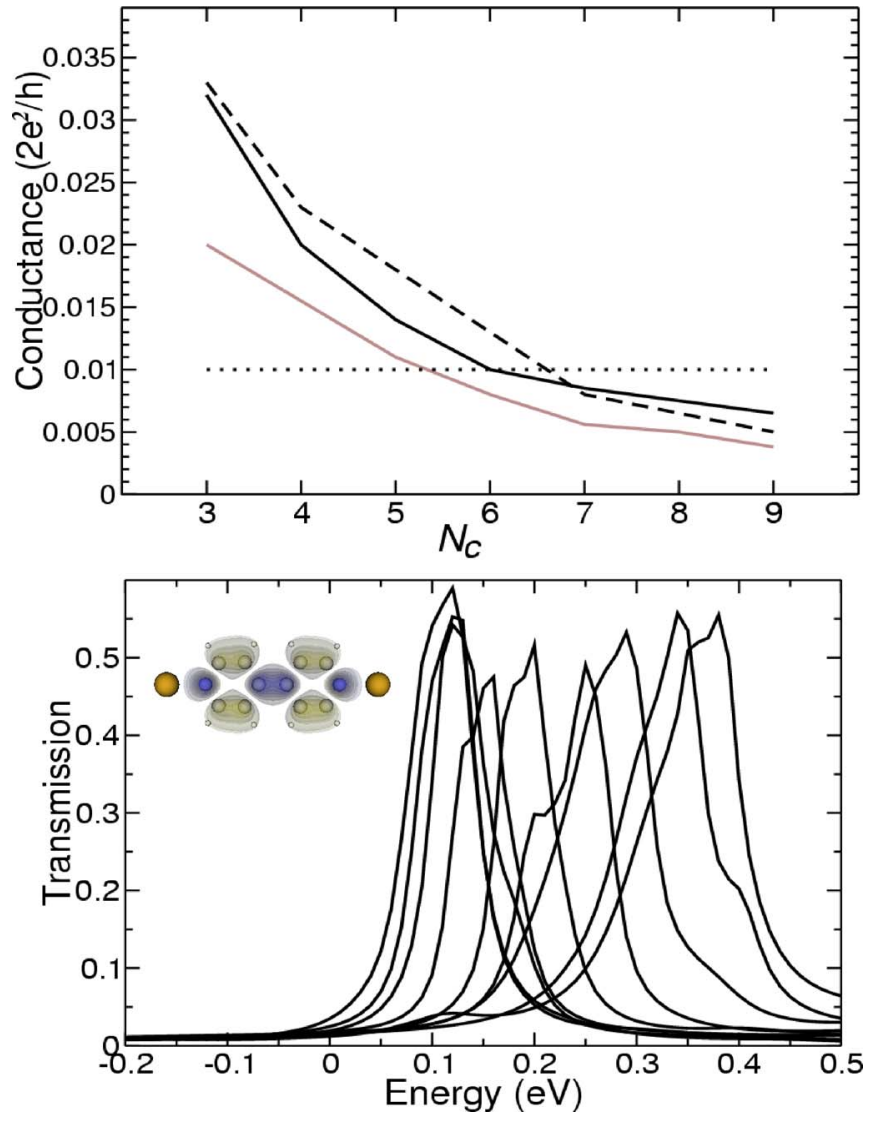

FIG. 3. (Color online) Top: Conductance in dependence on $N_{c}$ for torsion angles $\alpha=0^{\circ}$ (black line) and $25^{\circ}$ (gray line) in units of the conductance quantum $G_{0}=2 e^{2} / h$. The dashed line refers to a simple model explained in the text. The horizontal dotted line shows the experimental value. Bottom: Transmission functions for all nanojunctions $\left(\alpha=0^{\circ}\right)$. The peaks are due to the LUMO, shown in the inset, which rises continuously in energy as $N_{c}$ increases.

an appropriate transformation of the Kohn-Sham eigenstates. For the construction of these Wannier functions an energy range from the lowest lying orbital in the system up to $2 \mathrm{eV}$ above its Fermi energy has been chosen. Despite their optimal localization, they still have a fairly long-ranged but small tail, which for the systems studied in this article we truncate at a distance of $19.4 \AA$ from the center of each function. The couplings to the semi-infinite gold electrodes are included via self-energy matrices which are also represented in the Wannier function basis. A detailed discussion of the transport scheme can be found in Ref. 9. The central regions used in the transport calculation are the same as the supercells applied in the total energy calculations. These supercells contain $3 \times 3$ atoms in the directions perpendicular to the transport direction, and we have found that $\mathbf{k}$-point sampling in the transverse plane is important in order to obtain well converged results for the conductance. Thus all calculations presented in the following have been performed using a $4 \times 4$ k-point grid in the transverse Brillouin zone. For a systematic study of the role of $\mathbf{k}$-point sampling for transport calculations see Ref. 14.

In Fig. 3 we have plotted the calculated transmission near the Fermi level (which is taken as the zero point) for all geometries outlined in Fig. 1. The top panel of Fig. 3 shows the conductances for nanojunctions with $N_{c}=3$ to 9 for $\alpha=0^{\circ}$ and $\alpha=25^{\circ}$ as black and gray solid lines, respectively. The conductance is seen to vary significantly with the coordination number. However, the conductance for $\alpha=25^{\circ}$ is in good agreement with the experimentally observed value of one percent of the conductance quantum ${ }^{5} G_{0}=2 e^{2} / h$. It can be noted that this is the same range of $N_{c} \sim 5$, where also the forces in Fig. 2 match the measurements. But, as already discussed in connection with the force distributions, the actual propensity of the different structures in the experiment is not known.

We now proceed to analyze why the conductance varies the way it does with the coordination number. The analysis is based on the calculations with $\alpha=0^{\circ}$.

Within our calculational scheme it is possible to define molecular orbitals even when the molecule is coupled to the metal. The molecular orbitals are constructed from Wannier functions located on the molecule. ${ }^{9}$ It is therefore also possible to study the transport through a single or a subset of the molecular orbitals by simply removing the remaining orbitals from the basis set. When we focus on the contributions coming from individual molecular orbitals, we find that the single peak in the transmission function around $E_{F}$ (bottom panel in Fig. 3) is exclusively due to the lowest unoccupied molecular orbital (LUMO), whose wave function is shown in the inset. Since the peak is quite narrow for all geometries, correlation effects, leading to Coulomb blockade behavior in other weakly bonded molecular junctions, ${ }^{15}$ could be relevant for its accurate description. However, even in the "coherent limit" there is good agreement between our transport calculations and the experimental results.

One way of analyzing the shape of the $T\left(N_{c}\right)$ curve further is by applying a simple one-level model ${ }^{16}$ with only one molecular state (the LUMO) coupled symmetrically to the leads.

Within this model the transmission through a level at energy $E_{0}$ can be expressed as $T(\epsilon)=\Delta^{2} /\left[\left(\epsilon-E_{F}\right)^{2}+\Delta^{2}\right]$, where the peak width is defined by $\Delta=\pi V^{2} \rho_{g}^{0} . V$ is the coupling constant of the LUMO to its group orbital,,, 17 and $\rho_{g}^{0}$ is the density of states of its group orbital in the absence of coupling to the LUMO evaluated at $\epsilon$. By taking the values for $\rho_{g}^{0}, \epsilon$, and $V$ from the DFT calculation and using them to calculate $\Delta$, we obtain the dashed line in Fig. 3, which thus represents the conductance when the electrons are allowed to travel between the leads via the LUMO only. The good agreement of this one-level model when compared with our data further illustrates that the variation of the conductance is entirely controlled by the position of the transmission peak due to the LUMO and that the effect of the interference with other orbitals as well as through-vacuum tunneling are negligible.

But what is governing the LUMO position? We investigated the variation of the energetic position of the lowest lying molecular orbital (denoted MO1) with respect to the metal's Fermi level, in dependence on the distance between surface and molecule for all studied surface structures. In this analysis it can be seen that at the bonding distance, MO1 and the LUMO depend on $N_{c}$ in quantitatively the same way. This pattern cannot be explained in terms of vacuum level 
alignment alone, since the variation of the metal's work function with $N_{c}$ is parabolic with a minimum at $N_{c}=5-6$. However, MO1 is $\sim 10 \mathrm{eV}$ below the lowest lying Au valence states, and therefore the exchange or bonding interaction between the molecule and the surface do not have a direct influence on the energy of this orbital. From that we can conclude that the dependence of MO1 as well as the LUMO's energy on $N_{c}$ must be exclusively guided by rigid potential shifts, where we found that equilibrium charge transfer dominates at the optimal bonding distance and also Coulomb repulsion due to the confinement of electrons at the interface plays a role. We will discuss these electrostatic effects and their structure-dependent influence on the aligment of molecular levels in detail in a separate publication.

In summary, we presented a theoretical analysis of the forces and conducances of a bipyridine molecular junction. Since important parameters of the experiments, such as the atomic configuration of the electrode's surfaces are not known, we scanned a wide range of surface structures and found that both the forces and conductances depend crucially on their geometries, but our calculations agreed with the experiments in orders of magnitude for both quantities. The narrow peaks found in the statistics of the experiments, however, could not be directly reproduced from our calculations, and the statistics of the site-selectivity of the measurements of the adsorption of the molecules has to play a major role. Our study suggests that varying surface structures might be an important ingredient for a systematic understanding of the bonding and conductance properties of molecular junctions.

The Center for Atomic-scale Materials Physics is sponsored by the Danish National Research Foundation. We acknowledge support from the Nano-Science Center at the University of Copenhagen and from the Danish Center for Scientific Computing through Grant No. HDW-1101-05.
${ }^{1}$ C. Joachim, J. K. Gimzewski, R. R. Schlittler, and C. Chavy, Phys. Rev. Lett. 74, 2102 (1995).

${ }^{2}$ M. A. Reed, C. Zhou, C. J. Muller, T. P. Burgin, and J. M. Tour, Science 278, 252 (1997).

${ }^{3}$ J. Reichert, R. Ochs, D. Beckmann, H. B. Weber, M. Mayor, and H. v. Löhneysen, Phys. Rev. Lett. 88, 176804 (2002).

${ }^{4}$ R. H. M. Smit, Y. Noat, C. Untiedt, N. D. Lang, M. C. van Hemert, and J. M. van Ruitenbeek, Nature (London) 419, 906 (2002).

${ }^{5}$ B. Xu and N. J. Tao, Science 301, 1221 (2003); B. Xu, X. Xiao, and N. J. Tao, J. Am. Chem. Soc. 125, 16164 (2003).

${ }^{6}$ A. Bilić, J. R. Reimers, and N. S. Hush, J. Phys. Chem. B 106, 6740 (2002).

${ }^{7}$ S. Hou, J. Zhang, R. Li, J. Ning, R. Han, Z. Shen, X. Zhao, Z. Xue, and Q. Wu, Nanotechnology 16, 239 (2005); A. J. PerezJimenez, J. Phys. Chem. B 109, 10052 (2005).

${ }^{8}$ B. Hammer, L. B. Hansen, and J. K. Nørskov, Phys. Rev. B 59, 7413 (1999); S. R. Bahn and K. W. Jacobsen, Comput. Sci. Eng. 4, 56 (2002); The Dacapo code can be downloaded at http:// www.camp.dtu.dk/campos.
${ }^{9}$ K. S. Thygesen and K. W. Jacobsen, cond-mat/0501238, Chem. Phys. (to be published).

${ }^{10}$ J. P. Perdew, J. A. Chevary, S. H. Vosko, K. A. Jackson, M. R. Pederson, D. J. Singh, and C. Fiolhais, Phys. Rev. B 46, 6671 (1992).

${ }^{11}$ B. Hammer, L. B. Hansen, and J. K. Nørskov, Phys. Rev. B 59, 7413 (1999).

${ }^{12}$ M. Mavrikakis, P. Stoltze, and J. K. Nørskov, Catal. Lett. 64, 101 (2000); B. Hammer and J. K. Nørskov, Adv. Catal. 45, 71 (2000); S. R. Bahn, N. Lopex, J. K. Nørskov, and K. W. Jacobsen, Phys. Rev. B 66, 081405(R) (2002); Y. Xu and M. Mavrikakis, J. Phys. Chem. B 107, 9298 (2003).

${ }^{13}$ B. Hammer and J. K. Nørskov, Surf. Sci. 343, 211 (1995).

${ }^{14}$ K. S. Thygesen and K. W. Jacobsen, Phys. Rev. B 72, 033401 (2005).

${ }^{15}$ S. Kubatkin, A. Danilov, M. Hjort, J. Cornil, J. L. Brédas, N. Stuhr-Hansen, P. Hedegård, and T. Bjørnholm, Nature (London) 425, 698 (2003); O. Berman and S. Mukamel, Phys. Rev. B 69, 155430 (2004).

${ }^{16}$ D. M. Newns, Phys. Rev. 178, 1123 (1969).

${ }^{17}$ M. J. Kelly, Surf. Sci. 43, 587 (1974). 